\title{
Utility of Urinalysis as a Follow-up Surveillance Tool in Nonmuscle Invasive Bladder Cancer
}

\author{
Minuk Park, Bumjin Lim, Dalsan You, In Gab Jeong, Cheryn Song, Bumsik Hong, Choung-Soo Kim, \\ Hanjong Ahn, Jun Hyuk Hong
}

Department of Urology, Asan Medical Center, University of Ulsan College of Medicine, Seoul, Korea

Purpose: To evaluate the association between microscopic hematuria $(\mathrm{MH})$ detected by surveillance urinalysis and cancer recurrence in nonmuscle invasive bladder cancer (NMIBC) patients.

Materials and Methods: A total of 1,082 NMIBC patients who underwent transurethral resection of bladder tumor (TURB) procedures at Asan Medical Center between January 2017 and December 2019 were included. We retrospectively reviewed the follow-up data for these cases including cystoscopy, urinalysis, and urine cytology. The association between urine testing and cancer recurrence was assessed by both univariable and multivariable logistic regression analysis.

Results: The study patients had a median age of 68 years (interquartile range, $60-75$ years) and comprised 898 men and 184 women. Among the 1,428 TURB procedures conducted in this series, 548 of the lesions (38.4\%) were diagnosed as low-grade and $880(61.6 \%)$ as highgrade cancers. A total of 3,309 follow-up cystoscopies were conducted during the study period and were divided into high-grade (HG) (2,011 cases) and low-grade (LG) (1,298 cases) groups according to the latest TURB pathology. MH was found to have a statistically significant association with NMIBC recurrence in both the LG (odds ratio [OR], 1.57; 95\% confidence interval [CI], 1.107-2.223; $\mathrm{p}=0.011)$ and $\mathrm{HG}(\mathrm{OR}, 1.90 ; 95 \% \mathrm{Cl}, 1.434-2.517 ; \mathrm{p}<0.001)$ groups.

Conclusions: Urinalysis during follow-up may provide important information on cancer recurrence in NMIBC patients.

Key Words: Nonmuscle invasive bladder cancer, Follow-up, Urinalysis, Microscopic hematuria
Received August 9, 2021

Revised August 22, 2021

Accepted August 27, 2021

Corresponding author:

Jun Hyuk Hong

Email: jhhong@amc.seoul.kr https://orcid.org/0000-0003-2705-0481

\section{INTRODUCTION}

Bladder cancer ranked 10th in terms of global cancer incidence in 2020, totaling 573,000 cases worldwide. ${ }^{1}$ Painless gross hematuria $(\mathrm{GH})$ is the most common presenting symptom in bladder cancer and comprehensive evaluations including cystoscopy, upper tract imaging (computed tomography $[\mathrm{CT}]$ urography or magnetic resonance urography), and urine cytology are required in affected patients. When a bladder tumor is detected by an imaging modality or cystoscopy, transurethral resection of bladder tumor (TURB) is the standard and crucial treatment approach with pathological staging subsequently established through a histological evaluation of the resected tissue. ${ }^{2}$

Urothelial carcinoma accounts for about 90\% of all bladder cancers. These lesions are classified into 2 subtypes based on the depth of invasion i.e.. nonmuscle invasive bladder cancer (NMIBC) and muscle invasive bladder cancer (MIBC). NMIBC accounts for $75 \%$ of all bladder cancers at the 
initial diagnosis. ${ }^{2}$

In cases of MIBC, a radical cystectomy that is often preceded by neoadjuvant cisplatin-based chemotherapy offers the best chance for cure. In contrast, the treatment mainstay for NMIBC is a complete TURB paired with intravesical immunochemotherapy. Regular follow-ups which include cystoscopy, urine cytology, and upper tract imaging are also mandatory in these patients because of the high rate of bladder cancer recurrence, which can be as high as $80 \%$ within 5 years. ${ }^{3}$ Each follow-up modality has its own disadvantages however (e.g., discomfort and risk of infection with cystoscopy, risk of contrast reactions, and potential for radiationinduced cancers attributable to CT). Moreover, the natural course of NMIBC is heterogeneous with a variable risk of recurrence and progression. Hence, several risk grouping systems including the EORTC (European Organisation for Research and Treatment of Cancer) scoring system and the American Urological Association (AUA)/Society of Urologic Oncology risk stratification have been published to estimate the prognosis of individual patients. ${ }^{4,5}$ As such, the frequency, duration, and modality of the follow-ups should reflect each patient's degree of risk.

Many studies have already been conducted on the incidence of bladder cancer in patients with hematuria, with a reported occurrence of about $2.7 \%$ in microscopic hematuria $(\mathrm{MH})$ patients and $11 \%$ in $\mathrm{GH}$ patients. ${ }^{6-8} \mathrm{MH}$ cannot be overlooked however, especially in high-risk patients, and cystoscopy with upper tract imaging is recommended in these cases in accordance with AUA guidelines. ${ }^{9}$ The presence of $\mathrm{GH}$ in a patient diagnosed with bladder cancer was found to be a significant risk factor for cancer recurrence in a prior Korean study. ${ }^{10}$ Notably however, the association between $\mathrm{MH}$ found on urinalysis during follow-up and disease recurrence is not yet clear. Urinalysis with microscopy is a relatively affordable and noninvasive test for detecting $\mathrm{MH}$ that is readily available in the clinic. The aim of our present study was to establish the utility of $\mathrm{MH}$ as a predictive indicator for bladder cancer recurrence in NMIBC patients during followup.

\section{MATERIALS AND METHODS}

\section{Patient Population}

We retrospectively reviewed the medical records of 1,771 patients who underwent TURB at Asan Medical Center between January 2017 and December 2019. Surveillance data including cystoscopy, urinalysis with microscopy, and urine cytology results up to December 2020 were analyzed. The use of this database was approved by the Institutional Review Board (IRB) of Asan Medical Center (IRB number: 2021-0455).

The exclusion criteria for our patient cohort were as follows:

1. A diagnosis other than urothelial carcinoma.

2. A diagnosis of MIBC.

3. Had undergone ureteroscopy and nephroureterectomy for upper tract urothelial carcinoma (UTUC) during the study period.

\section{Incomplete TURB.}

\section{Diagnosis and Pathologic Evaluations}

AUA guidelines define $\mathrm{MH}$ as $\geq 3$ red blood cells per high power field on a microscopic evaluation of a single urine specimen and we utilized this definition in our present analyses. ' The TURB and urine cytology specimens were processed in accordance with standard pathology procedures, and slides were reviewed by expert genitourinary pathologists at our hospital. Cytology results were recorded as positive for those cases reported as 'high-grade urothelial carcinoma,' 'suspicious for high-grade urothelial carcinoma' or 'lower-grade 
urothelial neoplasia.' Any patients reported as 'negative for high-grade urothelial carcinoma' or with 'atypical urothelial cells' were considered to be negative, reflecting the Paris system. ${ }^{11}$ The TURB specimens were pathologically staged according to the American Joint Committee on Cancer staging 8th edition and graded in compliance with the 1973 and 2004/2016 World Health Organization grading systems. ${ }^{12,13}$

\section{Follow-up Protocols}

The postoperative surveillance protocol included cystoscopy, urinalysis, and urine cytology testing. Patients were generally examined every 3 months for the first 2 years postoperatively, every 6 months for the next 2 years, and annually thereafter. Recurrence was defined by the detection of a newly developed intravesical lesion on a cystoscope.

\section{Statistical Analysis}

Both univariable and multivariable logistic regression analysis were employed to evaluate the relevance between $\mathrm{MH}$ or urine cytology with bladder cancer recurrence. Correlations between cancer recurrence and assessed variables were expressed as an odds ratio (OR) with the 95\% confidence interval (CI). All statistical analyses were conducted using IBM SPSS Statistics ver. 25.0 (IBM Co., Armonk, NY, USA).

\section{RESULTS}

A total of 1,771 bladder cancer patients underwent TURB at our hospital between January 2017 and December 2019. Restaging TURBs were conducted based on the judgement of the surgeon. The 2nd stage TURBs were performed in instances where the first procedure was incomplete. If a tumor recurrence was evident by office cystoscopy during follow-up, another TURB or cold-cup biopsy was done. A total of 2,504 TURB procedures were conducted at our hospital during the study period.

We included only NMIBC cases in our current analysis and excluded 302 patients who were diagnosed with other diseases, such as cystitis, and 203 MIBC patients. We further excluded 183 patients with synchronous UTUC as MH can also be found in these cases. In cases of an incomplete resection, a 2nd stage TURB was done and any less invasive and lower grade cases were excluded from our final cohort. A total of 1,428 TURB procedures were done in the 1,082 NMIBC patients at our hospital during the observation period. Each TURB procedure was followed up with microscopic urinalysis, urine cytology, and cystoscopy as scheduled. If a urine test was not done with cystoscopy for any reason, we also precluded that particular case from the study cohort. A total of 3,309 follow-up cystoscopies that had been conducted on our study patients from 2017 to 2020 were reviewed, and these results were divided into 2 groups in accordance with the pathology of the previous TURB, i.e., low grade $(n=1,298)$ or high grade $(n=2,011)$ (Fig. 1). Table 1 summarizes the characteristics of our study subjects and the distribution of their bladder cancers by grade and stage. These included patients had a median age of 68 years (interquartile range [IQR], 60-75 years) and comprised 898 men and 184 women. The mean BMI ( \pm standard deviation) of these cases was $24.77 \pm 3.25$.

Following the 1,428 TURB procedures among the cases our current study series, 548 cases (38.4\%) were diagnosed with a low-grade tumor and the remaining 880 (61.6\%) with a high-grade cancer. During the surveillance period, there were 498 cases of bladder cancer recurrence in our present patient series. Table 2 indicates the relationship between $\mathrm{MH}$ and bladder cancer recurrence in the LG group in terms of the OR and $95 \%$ CI of 


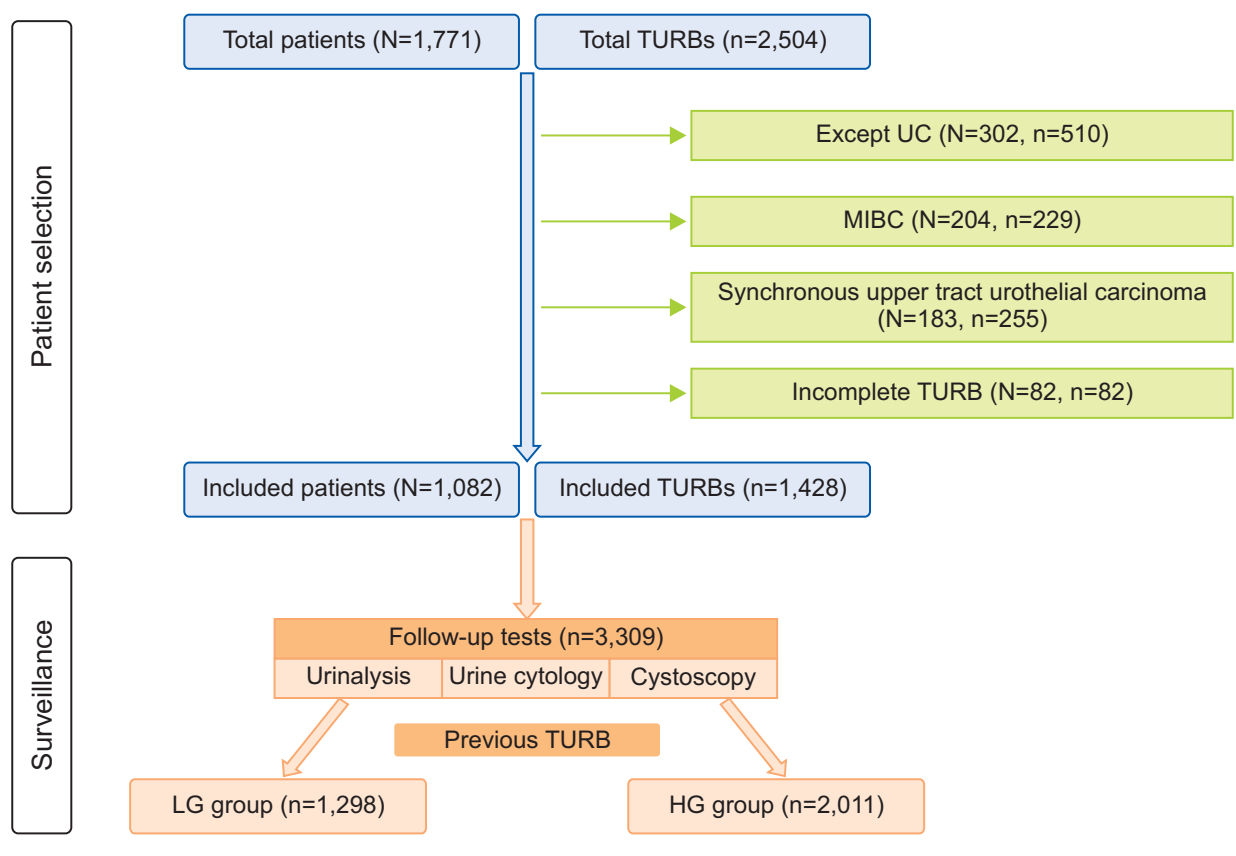

Fig. 1. Patient enrollment and grouping of follow-up cystoscopies according to the pathology of the previous TURB. TURB: transurethral resection of bladder tumor, UC: urothelial carcinoma, MIBC: muscle invasive bladder cancer, LG: low-grade, HG: highgrade.
Table 1. Patients and disease characteristics $(n=1,082)$

\begin{tabular}{lc}
\multicolumn{1}{c}{ Characteristic } & Value \\
\hline Age $(\mathrm{yr})$, median (IQR) & $68(60-75)$ \\
Sex & \\
Men & $898(83)$ \\
Women & $184(17)$ \\
Hypertension & $515(48)$ \\
Diabetes & $236(22)$ \\
BMI (kg/m $\left.{ }^{2}\right)$, mean \pm SD & $24.77 \pm 3.25$ \\
Intravesical BCG treatment & $480(44)$ \\
TURBs & 1,428 \\
Pathologic T stage & \\
Tis & $123(8.6)$ \\
Ta & $903(63.2)$ \\
T1 & $402(28.2)$ \\
Carcinoma in situ & $422(29.6)$ \\
2004 WHO grade & \\
Low grade & $548(38.4)$ \\
High grade & $880(61.6)$ \\
Follow-up tests & 3,309 \\
Positive findings & \\
Urinalysis & 714 \\
Urine cytology & 91 \\
Cystoscopy (cancer recurrence) & 498 \\
\hline
\end{tabular}

Values are presented as number (\%) unless otherwise indicated. IQR: interquartile range, BMI: body mass index, SD: standard deviation, BCG: Bacillus Calmette-Guérin, TURB: transurethral resection of bladder tumor, Tis: tumor in situ (only CIS without Ta or T1 lesion), WHO: World Health Organization. the OR (OR, 1.57; 95\% CI, 1.11-2.22; p=0.011). A positive finding on urine cytology showed no statistical association with the recurrence of an LG carcinoma. In the HG group, both $\mathrm{MH}$ and a positive urine cytology result were statistically associated with disease recurrence (OR, 1.84; 95\% CI, 1.38-2.44; $\mathrm{p}<0.001$ for $\mathrm{MH}$ and OR, 2.68; 95\% CI, 1.63-4.39; $\mathrm{p}<0.001$ for urine cytology). In our study cohort, 480 patients were treated with intravesical Bacillus Calmette-Guérin (BCG) therapy. Regardless of intravesical BCG therapy, $\mathrm{MH}$ and positive urine cytology were statistically associated with high cancer recurrence (OR, 1.59; 95\% CI, 1.18-2.15, $\mathrm{p}=0.003$ for $\mathrm{MH}$ and OR, 2.31; 95\% CI, 1.29-4.14; $\mathrm{p}=0.005$ for urine cytology in BCG treated group and OR, 1.59; 95\% CI, 1.162.16; $\mathrm{p}=0.004$ for $\mathrm{MH}$ and $\mathrm{OR}, 2.71$; $95 \% \mathrm{CI}, 1.23-$ 5.95; $\mathrm{p}=0.013$ in patients not treated with BCG) (Table 3).

\section{DISCUSSION}

Most urologists agree on the value of detecting $\mathrm{MH}$ when screening for bladder cancer in the 
Table 2. Univariable and multivariable logistic regression analysis for cancer recurrence in low-grade group and high-grade group

\begin{tabular}{|c|c|c|c|c|}
\hline \multirow{3}{*}{ Group } & \multicolumn{4}{|c|}{ Cancer recurrence } \\
\hline & \multicolumn{2}{|c|}{ Univariable } & \multicolumn{2}{|c|}{ Multivariable } \\
\hline & OR $(95 \% \mathrm{CI})$ & p-value & OR $(95 \% \mathrm{CI})$ & p-value \\
\hline \multicolumn{5}{|c|}{ Low-grade group } \\
\hline MH & $1.57(1.11-2.22)$ & 0.011 & & \\
\hline Cytology & Insignificant & 0.999 & & \\
\hline \multicolumn{5}{|c|}{ High-grade group } \\
\hline $\mathrm{MH}$ & $1.90(1.43-2.52)$ & $<0.001$ & $1.84(1.38-2.44)$ & $<0.001$ \\
\hline Cytology & $2.89(1.77-4.71)$ & $<0.001$ & $2.68(1.63-4.39)$ & $<0.001$ \\
\hline
\end{tabular}

OR: odds ratio, $\mathrm{CI}$ : confidence interval, $\mathrm{MH}$ : microscopic hematuria.

Table 3. Univariable and multivariable logistic regression analysis for cancer recurrence in patients treated with or without intravesical BCG therapy

\begin{tabular}{|c|c|c|c|c|}
\hline \multirow{3}{*}{ Group } & \multicolumn{4}{|c|}{ Cancer recurrence } \\
\hline & \multicolumn{2}{|c|}{ Univariable } & \multicolumn{2}{|c|}{ Multivariable } \\
\hline & OR $(95 \% \mathrm{CI})$ & p-value & OR $(95 \% \mathrm{CI})$ & p-value \\
\hline \multicolumn{5}{|l|}{ BCG group } \\
\hline MH & $1.66(1.23-2.24)$ & $<0.001$ & $1.59(1.18-2.15)$ & 0.003 \\
\hline Cytology & $2.56(1.44-4.55)$ & $<0.001$ & $2.31(1.29-4.14)$ & 0.005 \\
\hline \multicolumn{5}{|c|}{ Non-BCG group } \\
\hline MH & $1.59(1.17-2.18)$ & 0.003 & $1.59(1.16-2.16)$ & 0.004 \\
\hline Cytology & $2.76(1.26-6.06)$ & 0.011 & $2.71(1.23-5.95)$ & 0.013 \\
\hline
\end{tabular}

BCG: Bacillus Calmette-Guérin, OR: odds ratio, CI: confidence interval, MH: microscopic hematuria.

general population, especially in conjunction with a smoking history and old age. ${ }^{9}$ No data has yet been published however regarding the evaluation of the clinical performance of $\mathrm{MH}$ as a surveillance tool in NMIBC patients. In real-world clinical practice, clinicians usually employ urinalysis in the follow-up of NMIBC patients to check for $\mathrm{MH}$ as its presence can be a clue to cancer recurrence. To the best of our knowledge however, our current study represents the first attempt to assess the clinical value of $\mathrm{MH}$ in predicting an NMIBC recurrence.

We found in our present analysis that an $\mathrm{MH}$ detection on urinalysis during NMIBC surveillance is a statistically significant predictor for the recurrence of both LG and HG cancers. The OR of $\mathrm{MH}$ for recurrence was 1.57 (95\% CI, 1.11-2.22) in the LG group and 1.90 (95\% CI, 1.43-2.52) in the HG group. Meanwhile, a positive urine cytology showed an OR of 2.89 (95\% CI, 1.77-4.71) for recurrence in HG cancer, but no such significant association in LG cancer. Cystitis and hematuria are well-known complications of intravesical BCG therapy. ${ }^{14}$ So, subgroup analysis was also done to find out whether BCG treatment had affected our study results. However, MH was found to be statistically associated cancer recurrence not only in BCG untreated group but also in BCG treated group (Table 3 ).

The high rate of disease recurrence in NMIBC makes surveillance essential, including cystoscopy, urine cytology, and upper tract imaging. ${ }^{4}$ However, each of these modalities has distinct disadvantages. Although flexible cystoscopy has reduced the incidence of several complications associated with rigid cystoscopy, it is still invasive and uncomfortable for patients, and still confers some risk of a urinary tract infection. ${ }^{15,16}$ Also white light cystoscopy is limited in its ability to 
detect tiny papillary tumors and flat lesions such as CIS (carcinoma in situ). ${ }^{17}$ Urine cytology is an adjunctive tool during a NMIBC follow-up but has low sensitivity with regard to LG tumors. In addition, due to sampling limitations in terms of volume and cellularity, varying diagnostic yields from cytology have been noted. ${ }^{18}$ Our current study also found no relationship between a positive urine cytology test and LG cancer recurrence.

Many urine markers including NMP22, BTA, $\mathrm{uCyt}+$, UroVysion, etc. have been developed in an attempt to reduce or replace cystoscopy for surveilling NMIBC patients. However, no such marker at present has shown a sufficient level of sensitivity and specificity to achieve this and consequently, the current guidelines do not recommend their routine usage. ${ }^{19,20}$ Our present study was also conducted to gain further insights into possibly reducing the need for followup cystoscopies, and our data suggest that microscopic urinalysis could be an alternative approach in this regard.

A prior study has emphasized the efficacy of home-screening for $\mathrm{MH}$, reporting improvements in the survival rates for bladder cancer patients as a result of earlier detection in the screened population. ${ }^{21}$ Likewise, if $\mathrm{MH}$ is found on a urinalysis in a NMIBC patient before a scheduled follow-up clinicians will need to recommend early cystoscopy to check for cancer recurrence, especially in high risk patients. Moreover, $\mathrm{MH}$ found by a urinalysis that has been done immediately before cystoscopy could help clinicians interpret ambiguous findings such as an erythematous mucosal change.

Our current study had some limitations of note. First, as this was a retrospective study carried out at a single institution, we cannot exclude the probability of bias. For example, urinary tract infections could have affected the urinalysis results and such factor could not be thoroughly examined. Second, regardless of the results of urinalysis, all of our included patients routinely underwent follow-up cystoscopy. As such, we could not assess the effects of $\mathrm{MH}$ in both clinical practice and the prognosis of our NMIBC patients. Notwithstanding these limitations, however, our current study is the first to assess the utility of $\mathrm{MH}$ detection by urinalysis, one of the simplest and least expensive tests in a clinical setting, in the surveillance of large NMIBC cohorts for possible cancer recurrence. Further prospective studies with large sample populations are warranted to verify our research. We also recommend that urinalysis results should be carefully monitored when following up NMIBC patients.

\section{CONCLUSIONS}

$\mathrm{MH}$ appears to be a valuable indicator in the postoperative screening of NMIBC patients, regardless of the primary tumor grade. Further prospective studies are necessary to confirm this and elucidate the importance of detecting $\mathrm{MH}$ during NMIBC surveillance.

\section{CONFLICT OF INTEREST}

The authors claim no conflicts of interest.

\section{ORCID}

Minuk Park, https://orcid.org/0000-0002-5175-4000 Bumjin Lim, https://orcid.org/0000-0002-1746-6072 Dalsan You, https://orcid.org/0000-0001-8152-847X In Gab Jeong, https://orcid.org/0000-0003-4093-832X Cheryn Song, https://orcid.org/0000-0002-1823-4281 Bumsik Hong, https://orcid.org/0000-0003-1991-1229 Choung-Soo Kim, https://orcid.org/0000-0002-7464-3207 Hanjong Ahn, https://orcid.org/0000-0001-7608-5352 Jun Hyuk Hong, https://orcid.org/0000-0003-2705-0481 


\section{REFERENCES}

1. Sung H, Ferlay J, Siegel RL, Laversanne M, Soerjomataram I, Jemal A, et al. Global cancer statistics 2020: GLOBOCAN estimates of incidence and mortality worldwide for 36 cancers in 185 countries. CA Cancer J Clin 2021;71:209-49.

2. Babjuk M, Burger M, Zigeuner R, Shariat SF, van Rhijn BW, Compérat E, et al. EAU guidelines on non-muscle-invasive urothelial carcinoma of the bladder: update 2013. Eur Urol 2013;64:639-53.

3. Kamat AM, Hahn NM, Efstathiou JA, Lerner SP, Malmström PU, Choi W, et al. Bladder cancer. Lancet 2016;388:2796-810.

4. Chang SS, Boorjian SA, Chou R, Clark PE, Daneshmand S, Konety BR, et al. Diagnosis and treatment of non-muscle invasive bladder cancer: AUA/SUO guideline. J Urol 2016;196:1021-9.

5. Babjuk M, Burger M, Compérat EM, Gontero P, Mostafid AH, Palou J, et al. European Association of Urology Guidelines on non-muscle-invasive bladder cancer (TaT1 and carcinoma in situ) - 2019 update. Eur Urol 2019;76:639-57.

6. Davis R, Jones JS, Barocas DA, Castle EP, Lang EK, Leveillee RJ, et al. Diagnosis, evaluation and follow-up of asymptomatic microhematuria (AMH) in adults: AUA guideline. J Urol 2012;188(6 Suppl): 2473-81.

7. Tan WS, Sarpong R, Khetrapal P, Rodney S, Mostafid H, Cresswell J, et al. Can renal and bladder ultrasound replace computerized tomography urogram in patients investigated for microscopic hematuria? J Urol 2018;200:973-80.

8. Jubber I, Shariat SF, Conroy S, Tan WS, Gordon PC, Lotan $\mathrm{Y}$, et al. Non-visible haematuria for the detection of bladder, upper tract, and kidney cancer: an updated systematic review and meta-analysis. Eur Urol 2020;77:583-98.

9. Barocas DA, Boorjian SA, Alvarez RD, Downs TM, Gross CP, Hamilton BD, et al. Microhematuria: AUA/SUFU guideline. J Urol 2020;204:778-86.
10. Kim HS, Jeong CW, Kwak C, Kim HH, Ku JH. Novel nomograms to predict recurrence and progression in primary non-muscle-invasive bladder cancer: validation of predictive efficacy in comparison with European Organization of Research and Treatment of Cancer scoring system. World J Urol 2019;37:1867-77

11. Rosenthal DL, Wojcik EM, Kurtycz DF, editors. The Paris system for reporting urinary cytology. Cham (Switzerland): Springer International Publishing; 2016.

12. Magers MJ, Lopez-Beltran A, Montironi R, Williamson SR, Kaimakliotis HZ, Cheng L. Staging of bladder cancer. Histopathology 2019;74:112-34.

13. Moch H, Cubilla AL, Humphrey PA, Reuter VE, U1bright TM. The 2016 who classification of tumours of the urinary system and male genital organs-part a: renal, penile, and testicular tumours. Eur Urol 2016;70:93-105.

14. Lamm DL, van der Meijden PM, Morales A, Brosman SA, Catalona WJ, Herr HW, et al. Incidence and treatment of complications of bacillus CalmetteGuerin intravesical therapy in superficial bladder cancer. J Urol 1992;147:596-600.

15. van der Aa MN, Steyerberg EW, Sen EF, Zwarthoff EC, Kirkels WJ, van der Kwast TH, et al. Patients' perceived burden of cystoscopic and urinary surveillance of bladder cancer: a randomized comparison. BJU Int 2008;101:1106-10.

16. Yeung C, Dinh T, Lee J. The health economics of bladder cancer: an updated review of the published literature. Pharmacoeconomics 2014;32:1093-104.

17. Burger M, Grossman HB, Droller M, Schmidbauer J, Hermann G, Drăgoescu O, et al. Photodynamic diagnosis of non-muscle-invasive bladder cancer with hexaminolevulinate cystoscopy: a metaanalysis of detection and recurrence based on raw data. Eur Urol 2013;64:846-54.

18. VandenBussche CJ, Rosenthal DL, Olson MT. Adequacy in voided urine cytology specimens: the role of volume and a repeat void upon predictive 
values for high-grade urothelial carcinoma. Cancer Cytopathol 2016;124:174-80.

19. Shirodkar SP, Lokeshwar VB. Bladder tumor markers: from hematuria to molecular diagnostics--where do we stand? Expert Rev Anticancer Ther 2008;8:111123.

20. Xylinas E, Kluth LA, Rieken M, Karakiewicz PI, Lo- tan Y, Shariat SF. Urine markers for detection and surveillance of bladder cancer. Urol Oncol 2014;32: 222-9.

21. Messing EM, Madeb R, Young T, Gilchrist KW, Bram L, Greenberg EB, et al. Long-term outcome of hematuria home screening for bladder cancer in men. Cancer 2006;107:2173-9. 